\section{Effect of Topical Application of Phenylephrine Hydrochloride on Hyperplastic Gingivitis}

by

Clair L. Pernsteiner, D.D.S.*

MAJOR M. ASH JR. $\dagger$

IINFLAMMATORY HYPERPLASIA of the gingiva is a common clinical entity, being a response to injury by plaque and calculus. Removal of local irritants is the usual method of treating gingivitis; however, a solution containing phenylephrine hydrochloride (Phenodent Type A) has been used clinically to control hemorrhage and as a decongesting agent to control inflammatory hyperplasia.

Since oral mucosa varies in the type of surface epithelium and lamina propria, not all areas are equally penetrated by drugs. In general, those areas with a thin epithelial covering lying close to a rich capillary network will be most favorable for absorbing drugs. In the oral cavity, the buccal vestibule and sublingual mucosa rapidly absorb many drugs. ${ }^{1}$ Keratinized surfaces tend to be least absorptive. If the surface epithelium is lacerated or inflammed, the absorption of drugs is greatly enhanced. ${ }^{2}$

Although drugs applied to the mucosal surfaces may be absorbed into the systemic circulation, they are often employed for their local effect. Regardless of intent, drugs absorbed into the blood stream must be considered the same as if the drugs were absorbed parentally.

Very few vasoconstrictors other than epinephrine have been used topically in the oral mucosa; however, phenylephrine hydrochloride is used widely on many mucosal surfaces. ${ }^{3-5}$ It is used as a decongestant and hemostatic agent in the upper respiratory tract. Preparations using phenylephrine are also used as decongestants and hemostatic agents on the mucous membranes of the rectum. Phenylephrine is also used in ophthalmic preparations to decongest conjunctival mucous membranes.

At least one patent has been applied for claiming that a tablet containing chlorpheniramine maleate phenylephrine hydrochloride is capable of penetrating the oral mucosa to exert its therapeutic effect. ${ }^{1}$ No scientific evidence is available to substantiate this claim.

Clinically, it is apparent that phenylephrine hydro-

* Submitted in partial fulfillment of the requirements for the degree of Master of Science in Dentistry.

$\dagger$ Professor and Chairman, Department of Occlusion, The University of Michigan School of Dentistry, Ann Arbor, Mich 48104. chloride is a good decongestant and hemostatic agent that is capable of penetrating a variety of mucous membranes; however, little information is available concerning its effect on oral mucous membranes.

Phenodent Type $A \ddagger$ (brand of phenylephrine hydrochloride) is a mouth wash preparation employed for topical application to the oral mucous membranes. It is recommended for relief of congestion of the gingiva and oral mucous membranes, associated with gingivitis and post extraction swelling. The effectiveness of the latter, either as the sole treatment or as an adjunct to instrumentation, has not been evaluated. The purpose of this investigation was to evaluate the effectiveness of Phenodent Type A in the control of the gingival hyperplasia.

\section{Materials and Methods}

To evaluate the effect of Phenodent on gingival tissue, it was necessary to select patients with hyperplastic gingivitis. Only patients with a stable occlusion were selected; that is, no patients who exhibited abnormal clinical mobility of the teeth were utilized. Because local irritants have been shown to affect gingival inflammation, it was decided to score those factors which might be related to the degree of inflammation, viz., plaque, calculus, and crevice depth. Stone casts were chosen as the method by which changes in the gingival tissues could be directly measured. In order to evaluate changes in gingival contour, it was necessary to construct an instrument capable of precise orientation and measurement.

\section{Selection of Patients}

Fifty-two patients were selected from those who underwent initial screening examinations at The University of Michigan School of Dentistry, presenting the following characteristics: (1) Had not undergone orthodontic treatment in the past year; (2) Did not wear partial dentures; (3) No clinically detectable abnormal mobility of teeth; (4) At least 16 years of age; (5) No loss of contacts due to caries or extraction; (6) Was not to have restorative dentistry done during the study; (7) Sufficient number of teeth to mount on the template; (8) Patients had simple hyperplasia gingivitis and swelling on three or more teeth; (9) Not taking other drugs which might affect blood pressure or central stimulation; (10) No history of coronary heart disease, cardiovascular disease, or hyperthyroidism.

\section{Design of the Study}

The study was conducted under double blind conditions to avoid investigator bias. Three solutions $-\mathrm{a}$ placebo, a 0.25 and a $0.5 \%$ concentration of phenylephrine hydrochloride-were supplied for the study. The solutions were coded by someone not involved in the study. Matching of patients with solution was done according to a table of random numbers by the same person. As the patients were selected, they were di-

\footnotetext{
‡ Patent held by Karl Kosti, D.D.S., Royal Oak, Mich.
} 
vided into two groups. In Group I, the aim was to see if Phenodent $\mathrm{A}$ was capable of reducing gingival hyperemia. On the initial appointment a thorough evaluation of the patient's health history was conducted. The patient's blood pressure was recorded with a sphygmomamometer and a stethescope in an upright position. The degree of gingivitis, calculus, and pocket depth was scored using the Ramfjord Index, but for selected teeth and only for facial surfaces. ${ }^{6}$ Plaque was scored using the Kobayashi and Ash ${ }^{7}$ modification of the Ramfjord Index. Patients were told to continue their present oral hygiene regimen.

An impression was then taken with irreversible hydrocolloid* material and poured up immediately as described under "cast preparation". The patient was then given 15 to $25 \mathrm{cc}$ of solution as directed by the manufacturer. The blood pressure was again monitored 20 minutes later; however, no changes were found. Patients returned for the gingival, plaque, and pocket depth scoring and impressions were taken at 1, 3 and 6week intervals.

In Group II, the objective was to evaluate if the drug prevented the return of passive hyperemia over a 6week period. The procedure was the same as Group I except on the first day in addition to the procedures carried out for Group I, all teeth were thoroughly scaled and polished and a second impression was taken.

\section{Requirements of the Instrument}

In reviewing the literature, no instrument was described for direct gingival measuring which allowed for orientation of models and gingival measurements within a range of 0.001 inches. Therefore, it was decided to construct an instrument for this purpose. Before constructing the instrument, the following objectives were outlined: (1) Orientation of all models of the same mouth within 0.001 inch accuracy in three planes; (2) Convenient method of mounting models; (3) All teeth on interdental papilla should be accessible to measurement; (4) Measuring should be obtainable from undercuts and narrow interdental papillae; (5) Measuring should be easy and not time consuming; (6) Construction of the instrument should be inexpensive.

\section{Description of the Instrument}

The instrument consists of a bench model drill press, a mandrel with a fixed and removable collar, corner angle, an $x-y$ traverse, a 125 pound magnetic hold down, a template, a dial height gauge and four dial indicator gauges with 0.001 -inch graduations (Fig. 1). A 0.025 -inch stylus, made from instrument steel was used to replace the pointer on each gauge, so that small areas were accessible. All gauges conformed to A.G.D. specifications. All milled or ground surfaces were accurate to 0.001 inch across the entire surface.

A template of $3 \frac{1 / 2}{2} \times 3 \frac{1 / 2}{1 / 4}$ inch $( \pm 0.0005$ inch $)$

\footnotetext{
* Jeltrate, H. B. Caulk Company.
}

dimensions was milled. A hole measuring 0.375 inch in diameter was bored in the center of the template so that it could be positioned on the mandrel. Three additional holes were drilled in the template so that the models could be seated on the template. The drill press served to hold the mandrel, raise and lower the model, and provide a flat surface to which a base plate was mounted. The base plate consisted of a $13 \times 16 \times 1 / 2$ inch plate of steel. The broad surfaces of the steel plate were ground flat on a surface grinder and then fixed to the table of the drill press. A corner angle with lugs was constructed and secured to the base plate with thumb screws; its purpose was to guide the template as it moved vertically to prevent any rotation of the models on the mandrel and to attach the dial indicator used for vertical measurement. The $x-y$ traverse was mounted to the base plate and served as a mount for one of the dial indicator gauges. It permitted travel in two directions so that several interdental papillae could be measured. The magnetic hold down was also used to secure a dial indicator gauge. Three 2-inch and one 1-inch travel (0.001 inch accuracy) dial indicator gauges were used to orientate and measure changes on the model. They were numbered I, II, III, and IV respectively. Gauge I was mounted on the corner angle and was used for vertical orientation of the template and changes in vertical position of the template as the models were raised or lowered to the measurement sites. Gauge II

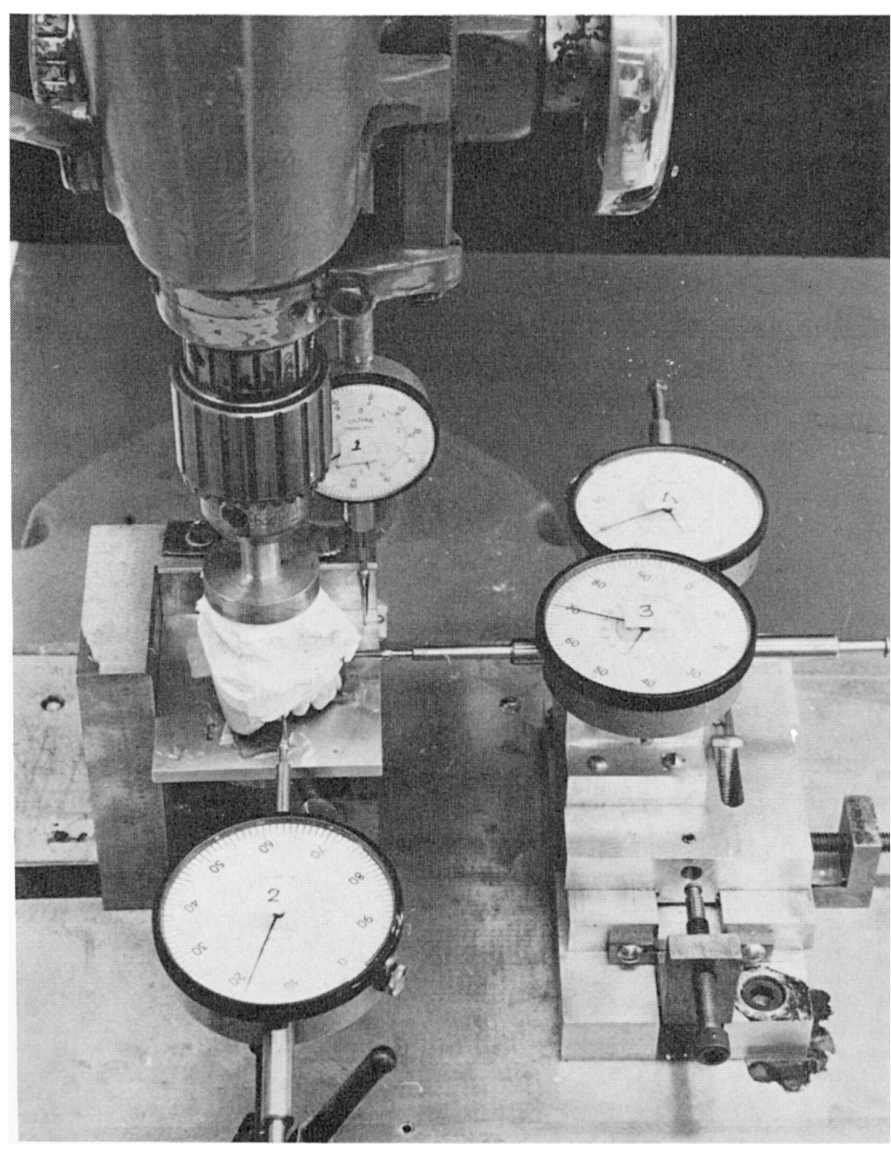

FIGURE 1. Instrument with mounted model. Gauges are numbered 1 to 4 respectively. 
TABLE 1. Analysis of Variance-Comparing Mean PDI Variable Scores Among Solutions for Combined Interdental Papillae Affected by Teeth 6-11, 22-27, Group I

\begin{tabular}{|c|c|c|c|c|c|c|c|c|c|c|}
\hline \multirow[b]{2}{*}{ PDI variable } & \multirow[b]{2}{*}{ Time* } & \multicolumn{6}{|c|}{ Adjusted mean PDI scores } & \multirow{2}{*}{$\begin{array}{c}\text { Total } \\
n \ddagger\end{array}$} & \multirow[b]{2}{*}{ Probability } & \multirow[b]{2}{*}{ Significance } \\
\hline & & $0.5 \% \mathrm{PE} \dagger$ & $n \ddagger$ & Control & $n \ddagger$ & $\begin{array}{c}0.25 \% \\
\mathrm{PE} \dagger\end{array}$ & $n \ddagger$ & & & \\
\hline Gingivitis & 2 & -0.29 & 8 & -0.40 & 7 & -0.22 & 8 & 23 & 0.805 & ns $\S$ \\
\hline Plaque & 2 & -0.05 & 8 & -0.32 & 7 & -0.49 & 8 & 23 & 0.272 & ns \\
\hline Calculus & 2 & Not scored & & & & & & & & \\
\hline Crevice depth & 2 & 0.01 & 8 & -0.21 & 7 & -0.21 & 8 & 23 & 0.264 & ns \\
\hline Gingivitis & 3 & -0.57 & 6 & -0.64 & 5 & -0.59 & 7 & 18 & 0.954 & ns \\
\hline Plaque & 3 & -0.05 & 6 & -0.16 & 5 & -0.58 & 7 & 18 & 0.342 & ns \\
\hline Calculus & 3 & Not scored & & & & & & & & \\
\hline Crevice depth & 3 & -0.05 & 6 & -0.10 & 5 & -0.21 & 7 & 18 & 0.825 & ns \\
\hline Gingivitis & 4 & -0.29 & 8 & -0.66 & 7 & -0.64 & 8 & 23 & 0.334 & ns \\
\hline Plaque & 4 & -0.47 & 8 & -0.21 & 7 & -0.94 & 8 & 23 & 0.045 & yes \\
\hline Calculus & 4 & -0.13 & 8 & -0.57 & 7 & -0.14 & 8 & 23 & 0.621 & ns \\
\hline Crevice depth & 4 & -.09 & 8 & -0.22 & 7 & -0.32 & 8 & 23 & 0.382 & ns \\
\hline
\end{tabular}

${ }^{*}$ Number of weeks after initiation of study.

$\dagger \mathrm{PE}=$ phenylephrine solution.

$\ddagger n=$ number of subjects in each group.

$\S \mathrm{ns}=$ nonsignificant.

was mounted on the magnetic hold down used only for horizontal orientation of the teeth. Gauge III was fixed to the $x-y$ traverse and was used for profile orientation and for measurement of the interdental papilla. Gauge IV was fixed to the base plate and indicated the changes in position of the $x-y$ traverse from one interdental papilla to another in a horizontal plane. The dial height gauge was used to measure the distance between template and tips of the teeth.

\section{Accuracy of the Instrument}

To test the accuracy of the instrument, three pilot studies were performed. The first study had to do with how accurately three models of the same mouth would fit into the autopolymerizing acrylic seats. Three models each of three different mouths were made from impressions taken at the same sitting. One model from each patient was mounted to the template with self curing acrylic. $\dagger$ The first molar and incisors were chosen as seating teeth in each case. The cuspids and second bicuspids were chosen for measurement. The distance from the tip of these teeth to the template on both the right and left side were measured with 0.001 inch precision ground feeler gauges. After this procedure was completed for the original model it was repeated for the two remaining models. The results are presented in Table 1.

\section{Preparation of the Casts and Mounting Casts on the Instrument}

After the impressions were taken they were poured up with model stone $\ddagger$ according to the manufacturer's directions. The water powder ratio was the same for each impression. The teeth and gingiva and the palate

$\dagger$ Duralay Reliance Dental Mfg. Co., Chicago, Ill.

$\ddagger$ Velmix, Kerr Dental Mfg. Co., Romulus, Michigan or floor of the mouth area of the impression were covered with stone; however, a hole was left near the anterior of the impression. After hardening, the impression was trimmed of excess stone and mounted on the instrument. The teeth were seated in the template. The template was placed on the mandrel and the removable collar was fastened in position. After tapping the template and the model against the secured removable collar, No. 2 plaster was placed in the space between the model and the fixed collar. The model was removed after the plaster hardened. The same procedure was done for the remaining models of the same patient.

\section{Measurement Procedure}

After preparation of the models of the same patient for orientation on the instrument, the measurement procedure was performed in the following manner. The vertical position of the model was adjusted and recorded so that all models of the same mouth would be measured from the same vertical position. The corner angle was positioned against the template. The dial height gauge was then used to determine the distance between three teeth tips and the template so that any error in fitting the teeth into the acrylic seats could be noted. Next, the Number II and III gauges were placed against a particular tooth chosen for a reference point. The position of the teeth chosen for orientating the models correctly in all planes was recorded for each tooth.

After orientation of the models, Gauge III was traversed to the first interdental papilla to be measured. The position of Gauge III was recorded from Gauge IV. The vertical position was adjusted so that the summit of the papilla was placed in a position to measure. The position where the stylus touched the interdental papilla of the model was recorded. Recordings were 
476 Pernsteiner, Ash

made at 0.025 -inch intervals for five successive points. The gauge was then moved to the adjacent papilla and the process was repeated. This procedure was repeated for the models taken at subsequent times.

\section{RESULTS}

The hypothesis in this study was that all solutions were equal. The customary significance levels $(0.05$ and 0.01 ) were used to reject or accept the null hypothesis.

The results of the comparison of mean periodontal disease index variables among solutions combining all tooth surfaces which affect the interdental papillae (6$7,22-27)$ are summarized for Group I in Table 1 and for Group II in Table 2. There was no significant difference among the solutions tested.

\section{Discussion}

The mean PDI scores for individual tooth surfaces affecting an interdental papilla were analyzed. Since no pattern of significance for any of the solutions was established, it was decided to analyze the combined mean of all teeth affecting the interdental papillae for gingivitis, plaque, calculus, and pocket depth for both Groups I and II. The mean was also analyzed for the upper and lower teeth separately for both groups. The results again did not show a pattern of significance for any of the solutions. A statistically significant reduction was noted for plaque values in time 4 in Group I, but was numerically small and of limited biologic significance. There was no suggestion by the manufacturer that the solution would reduce plaque. There was a statistically significant reduction in pocket depth at time 4 in Group II; however, the greatest reduction was in Solution II which was the control.

Because of the small sample size and the relatively large variance between the samples it was not possible to determine if the active agents may have had a small effect. The data does not suggest that an increase in the sample size would show such effectiveness.

The adjusted means of cast measurements were then covaried with gingivitis among the three solutions to evaluate if reduction occurred between time 1 and time 4 on the labial surface of the interdental papillae. Again, no significant reduction pattern was established.

The amount of phenylephrine hydrochloride contained in the solutions appeared to be well tolerated systemically by all but one subject. No clinical signs of local irritation or abnormalities were noted on any of the subjects using any of the solutions.

The accuracy limitation of the instrument was 0.001 inch. There was no problem in reproducibility. Measurements on the same cast were accurately repeated within \pm 0.001 inch of variation; however, great care had to be taken to avoid small pieces of debris causing errors in repositioning. Casts made from impressions taken at the same sitting fit the template well; however, some casts taken at the 1,3, and 6-week interval did not seat as accurately. The instrument was not designed to measure which teeth may have moved. The addition of water to the cast once the stone had completed setting did not change the dimensional stability of the models. Upon thorough drying, the models maintained their original dimension.

The solutions tested were not effective in reducing gingival hyperplasia and, therefore, would be of questionable clinical significance. The instrument designed for measuring changes in tissue dimension proved to be accurate and effective and may be used in future studies to measure the effect of various procedures and/or medicaments on tissue contour.

\section{SumMary}

A double blind study was conducted to evaluate the effectiveness of Phenodent Type A (brand of phenylephrine hydrochloride) on decongesting hyperplastic

TABLE 2. Analysis of Variance-Comparing Mean PDI Variable Scores among Solutions for Combined Interdental Papillae Affected by Teeth 6-11, 22-27, Group II

\begin{tabular}{|c|c|c|c|c|c|c|c|c|c|c|}
\hline \multirow{2}{*}{ PDI variable } & \multirow{2}{*}{ Time* } & \multicolumn{6}{|c|}{ Adjusted mean PDI scores } & \multirow{2}{*}{$\begin{array}{c}\text { Total } \\
n \ddagger\end{array}$} & \multirow{2}{*}{ Probability } & \multirow{2}{*}{ Significance } \\
\hline & & $0.5 \% \mathrm{PE} \dagger$ & $n \ddagger$ & Control & $n \ddagger$ & $0.25 \% \mathrm{PE}^{*}$ & $n \ddagger$ & & & \\
\hline Gingivitis & 2 & -0.49 & 6 & -0.96 & 7 & -0.88 & 7 & 20 & 0.108 & ns§ \\
\hline Plaque & 2 & -0.41 & 6 & -1.02 & 7 & -1.07 & 7 & 20 & 0.580 & ns \\
\hline Calculus & 2 & -0.77 & 6 & -1.26 & 7 & -0.87 & 7 & 20 & 0.434 & ns \\
\hline Crevice depth & 2 & -0.38 & 6 & -0.33 & 7 & -0.34 & 7 & 20 & 0.930 & ns \\
\hline Gingivitis & 3 & -0.55 & 7 & -0.80 & 7 & -0.82 & 7 & 21 & 0.456 & ns \\
\hline Plaque & 3 & -0.19 & 7 & -0.61 & 7 & -0.59 & 7 & 21 & 0.576 & ns \\
\hline Calculus & 3 & -0.64 & 7 & -0.91 & 7 & -0.78 & 7 & 21 & 0.692 & ns \\
\hline Crevice depth & 3 & -0.31 & 7 & -0.34 & 7 & -0.31 & 7 & 21 & 0.942 & ns \\
\hline Gingivitis & 4 & -0.50 & 7 & -0.62 & 8 & -0.56 & 7 & 22 & 0.863 & ns \\
\hline Plaque & 4 & -0.12 & 7 & -0.35 & 8 & -0.89 & 7 & 22 & 0.452 & ns \\
\hline Calculus & 4 & -0.59 & 7 & -0.94 & 8 & -0.76 & 7 & 22 & 0.549 & ns \\
\hline Crevice depth & 4 & -0.13 & 7 & -0.43 & 8 & -0.17 & 7 & 22 & 0.016 & yes \\
\hline
\end{tabular}

* Number of weeks after initiation of study.

$\dagger \mathrm{PE}=$ phenylephrine solution.

$\ddagger n=$ number of subjects in each group.

$\S \mathrm{ns}=$ nonsignificant. 
gingivitis. Three solutions were used: a $0.5 \%$ a placebo, and a $0.25 \%$ concentration of phenylephrine hydrochloride. The periodontal disease index was used to score variables which might have an effect on gingival response to local irritants. Impressions were taken and casts were made on 45 subjects at $0,1,3$, and 6week intervals. An instrument with accuracy of 0.001 inch was constructed to measure the changes in the interdental papillae of the stone casts. No significant reduction of gingival volume was established for any of the three solutions.

\section{CONCLUSIONS}

1. A mouthwash (Phenodent, Type A) containing $0.5 \%$ phenylephrine hydrochloride is not effective for the control of simple inflammatory gingival hyperplasia over a period of 1 to 6 weeks.

2. A mouthwash (Phenodent, Type A) containing $0.25 \%$ phenylephrine hydrochloride is not effective for the control of simple inflammatory gingival hyperplasia over a period of 1 to 6 weeks.

\section{ACKNOWLEDGMENTS}

We express our thanks to: Mr. Dallas Garrett and Peter Wiernick for assistance in design and preparing the measuring instrument, Miss Sally Holden for her preparation of the computer analysis, and Dr. Karl Kosti for financial assistance.

\section{BIBLIOGRAPHY}

1. Gibaldi, M., and Kaniq, J. L.: Absorption of drugs through the oral mucosa..I Oral Ther Pharm 1: 440, 1965.

2. Accepted Dental Therapeutics, 1969-1970, ed 33, pp 98-101, American Dental Association, 1968.

3. Goodman, L. S., and Gilman, A.: The pharmacological basis of therapeutics, ed 4, pp 485-515. New York, Macmillan, 1970.

4. Osol, A., Pratt, R., and Altschule, M. D.: The United States dispensatory and physicians pharmacology, ed 2, p 896. Philadelphia, J. B. Lippincott Co., 1967.

5. Physician's Desk Reference to Pharmaceutical Specialties and Biolgicals, ed 25, Oradell, N.J., Med. Economics, Inc., 1971.

6. Ramfjord, S. P.: Indices for prevalence and incidence of periodontal disease. J Periodontol 30: 51, 1959.

7. Koboyashi, L. Y., and Ash, M. M., Jr.: A clinical evaluation of an electric toothbrush used by orthodontic patients. Angle Orthod 34: 709, 1964.

\section{Abstracts}

An Engineering Analysis of Oral Irrigators with Respect to Their Washing Ability

Liljestrand, W. E.

J Am Soc Prev Dent 6: 23, July/August, 1976.

Two factors must be considered in the process of washing teeth: a) the way the water stream applies forces to the cleaning area, and b) limitations which control the hole diameter in the spray tip. The main characteristics of an open stream type of irrigator are velocity, diameter, and direction. The amount of force on the tooth or soft tissue surface depends on the velocity of the end of the stream in contact with the surface. With spray from an electric pump, the size of the supply tank, motor and pump as well as the size of the hole in the tip, are factors. However, the different tip hole sizes of $1.5 \mathrm{~mm}$ and 1.0 $\mathrm{mm}$ may be important in relation to the area covered. 7851 Metro Parkway, Suite 301, Minneapolis, Minn 55420.

Plaque-Removing Effect of Dental Floss and Toothpicks in Children 2 to 13 Years of Age

Anaise, J. Z.

Comm Dent Oral Epidemiol 4: 137, July, 1976.

To compare the plaque-removing effect of dental floss and toothpicks on interproximal surfaces of teeth, 21 children between the ages of 12 and 13 years were divided into three groups of seven each. The modified patient hygiene performance index (PHPI) was used at the beginning and the end of the study to evaluate plaque. Group I used waxed dental floss twice daily, Group II used aspen toothpicks (triangular) twice daily with specific instructions, and Group III was a control group using no device. The results showed that dental floss had a greater effect in removing plaque from the interproximal areas, than toothpicks. School of Dental Medicine, Hadassah Medical Center, P.O. Box 499, Jerusalem, Israel.

Dr. Teresa Toledo
Quantity, Nature, and Time Required for Recurrence of the Microbial Mass Irrigated from the Periodontal Pocket

Killoy, J. W.

J Am Soc Prev Dent 6: 17, July/August, 1976.

To observe the physical and microscopic characterisics of the microbial aggregates following repeated irrigation, samples were obtained from three patients at 1- to 7-day intervals following the initial irrigation. The periodontal pocket of one patient was irrigated at additional 8-, 14-, and 25-day intervals to determine if the repeated irrigation had affected the recurrence of the material. Irrigation produced a reduction in the volume of contents flushed from the pocket which persisted in patient B up to 25 days. Department of Periodontics, Wilford Hall, USAF Medical Center, Lackland AFB, Texas.

Dr. Eduardo Stein

A New Model for the Study of Transport of $14 \mathrm{C}$ Diphenylhydantoin through the Gingival Crevicular TISSUES IN THE RABBIT

Steinberg, A. D., Allen, P., and Jeffay, H. Arch Oral Biol 20: 865, December, 1975.

After intravenous injection of DPH (diphenylhydantoin) gingival samples were analyzed to determine tissue levels in the free and attached gingiva of six rabbits. By monitoring the passage of a radioactive tracer $14_{\mathrm{C}}$ Diphenylhydantoin (DPH) through the crevice, into the tissue and then into the blood, it was noted that after 2 hours, substantial amounts were found in the crevicular tissues. Intravenous administration of $14_{\mathrm{C}} \mathrm{DPH}$ revealed that after 2 hours, drug concentration in the crevicular tissue and total gingival concentrations were not significantly different. Department of Periodontics, School of Dentistry, and Department of Biochemistry, School of Medicine, University of Illinois, Chicago, Ill 60612.

Dr. Jacob Saad 